\title{
Developing Software for Simulation of Material and Wave Properties of Piezoelectromagnetics Incorporating Gravitational Phenomena
}

\author{
Aleksey Anatolievich Zakharenko \\ International Institute of Zakharenko \\ Waves (IIZWs, www.iizw.ru) \\ Krasnoyarsk, Russia \\ http://orcid.org/0000-0003-2677-4904
}

\begin{abstract}
This short report acquaints the reader with the developed software that can work with piezoelectrics, piezomagnetics, and piezoelectromagnetics (PEMs) with incorporation of gravitational phenomena, i.e. piezogravitotorsionoelectromagnetic (PGTEM) materials. This software can calculate the material properties and wave characteristics in all the aforementioned continuous media. Also, it allows the PEM and PGTEM composite creation from the material parameters of both piezoelectrics and piezomagnetics that present in the software database or can be loaded from a file. The software interface and sample calculations are demonstrated.
\end{abstract}

Keywords-software developing, simulation of material and wave properties, magnetoelectric and gravitational effects, acoustic wave propagation, piezoelectromagnetics

\section{INTRODUCTION}

The theory on the acoustic wave propagation coupled with the electrical, magnetic, gravitational, and torsional (i.e. cogravitational) potentials was developed in 2016 [1] and 2017 [2]. Some mathematical problems were resolved in 2018 [3]. It was found that these four-potential shear-horizontal (4P-SH) acoustic waves can propagate along the surface of the suitable solid [1], along the common interface between two dissimilar solids [4], and in plates [5].

All the obtained speeds of the different 4P-SH acoustic waves depend on the material parameter called the coefficient of the electromagnetogravitotorsionomechanical coupling (CEMGTMC) discussed in [6]. In the solids, these acoustic wave speeds can also depend on the speeds of the electromagnetic and gravitational (namely gravitotorsional) waves, and two faster speeds. These two fast speeds can exist in solids and a vacuum. Therefore, these fast waves can be used for instant interplanetary (interstellar and even intergalactic) communications. Review [7] has combined evaluations of propagation speeds of gravitational phenomena in continuous media that was done from Newton's time to this century because Newton's theory of gravitation assumes an instant speed for gravitational phenomena. Some suggestions concerning the instant interplanetary communication were introduced in [8]. For this purpose, the development of proper infrastructure on the Earth is necessary. It is obvious that the successful development can be based on resolving many theoretical, mathematics, experimental, and engineering problems.

First of all, it is necessary to investigate these problems for solid continua. Then, it is possible to use obtained experience for a vacuum. However, acoustic waves cannot propagate in a vacuum. To study the acoustic wave propagation in the piezoelectromagnetic (i.e. magnetoelectric) materials with taking into account the gravitational phenomena, i.e. the piezogravitotorsionoelectromagnetic (PGTEM) materials, is significantly more complicated in comparison with the purely piezoelectromagnetic case. As a result, many material parameters must be taken into account for calculation of wave parameters. To put forward investigations of these PGTEM (composite) materials, the interactive software was created at the International Institute of Zakharenko Waves (IIZWs, www.iizw.ru).

The following section introduces the developed software. This software deals with all the aforementioned materials because the study of the PGTEM materials requires this peculiarity. For this aim, it is necessary to use existing or created piezoelectrics, piezomagnetics, and piezoelectromagnetics, as well as auxiliary piezogravitics, piezotorsionics, and piezogravitotorsionics in order to create a PGTEM material with desired properties.

\section{DESCRIPTION}

The created software (first version) written with the free Lazarus tools can be downloaded here: https://www.researchgate.net/publication/338517269 On ev aluations of fast speeds of propagation of gravitational $p$ henomena_A review as a file.zip that contains the single file.exe $(\sim 10 \mathrm{MB})$ for installation on a Windows $7 / 8 / 10$ computer. For installation, it requires only $\sim 60 \mathrm{MB}$ of the hard disk space and can be uninstalled similar to any other software. After installation, the main form shown in figure 1 starts. This form has two TabSheets shown in figures 1 and 2, respectively.

For user reference, the first TabSheet provides the vacuum parameters just below the main title. The electric, magnetic, gravitic constants and the speed of light can be found in the reference book on physics [9]. Using the gravitic constant and the speed of light, the torsionic constant can be calculated. The other vacuum parameters in figure 1 have the evaluated values borrowed from $[3,4,6]$. For convenience, all the vacuum parameters have their physical dimensions. These vacuum parameters are used in suitable calculations of speeds of the acoustic waves propagating in the solids when there is a coupling with a vacuum. Below the vacuum parameters there are dialogs for selection of suitable materials. First of all, it is necessary to choose the crystal symmetry, hexagonal or cubic. Each crystal symmetry loads its own default list of material parameters for piezoelectrics, piezomagnetics, piezoelectromagnetics, piezogravitics, piezotorsionics, and piezogravitotorsionics. As soon as the user has chosen any of the aforementioned materials the results of the calculations are shown in two output areas located at bottom-right of the main form shown in figure 1 . 


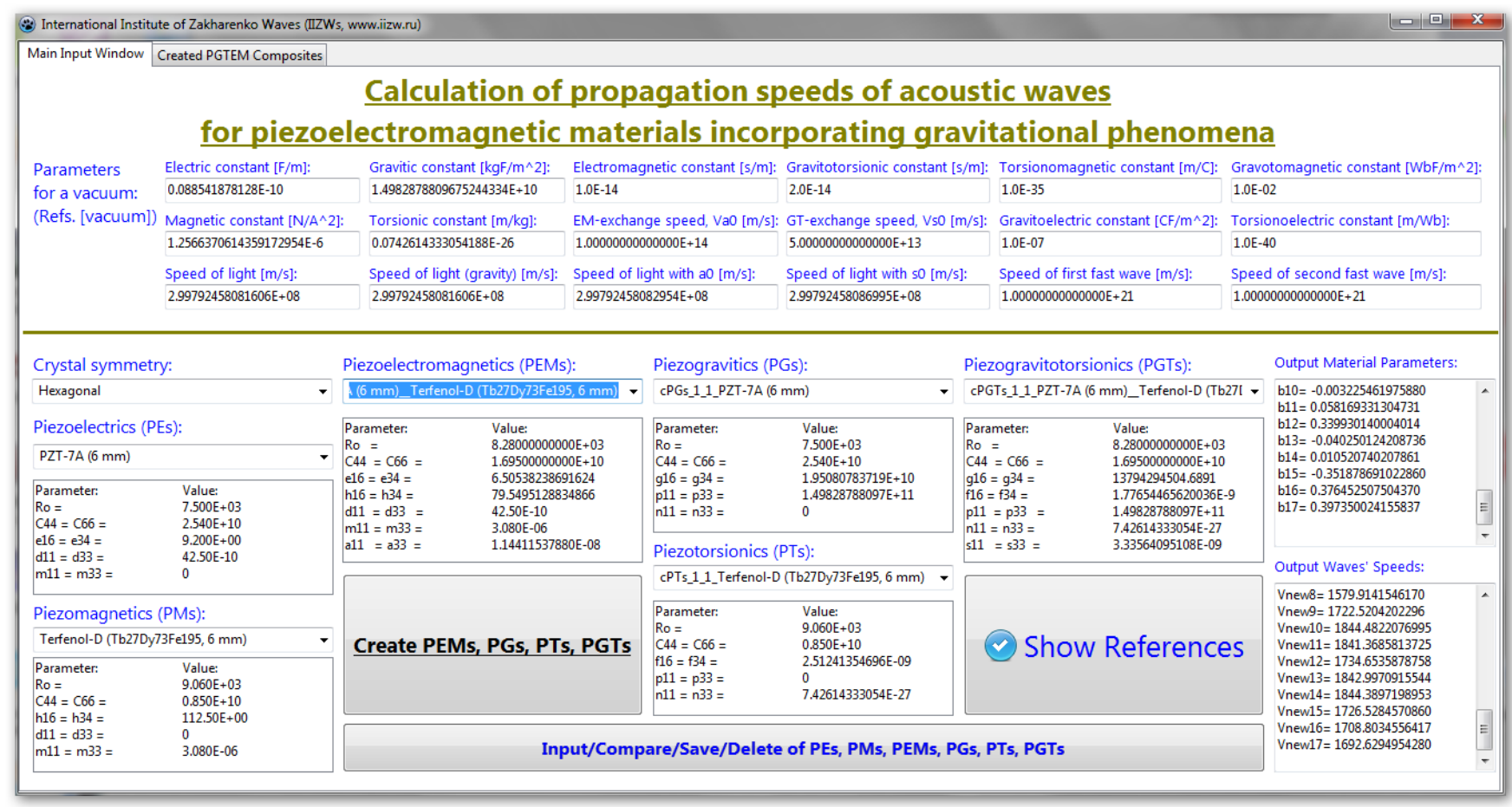

Fig. 1. The main form that starts as soon as this software is installed on a Windows 7/8/10 computer.

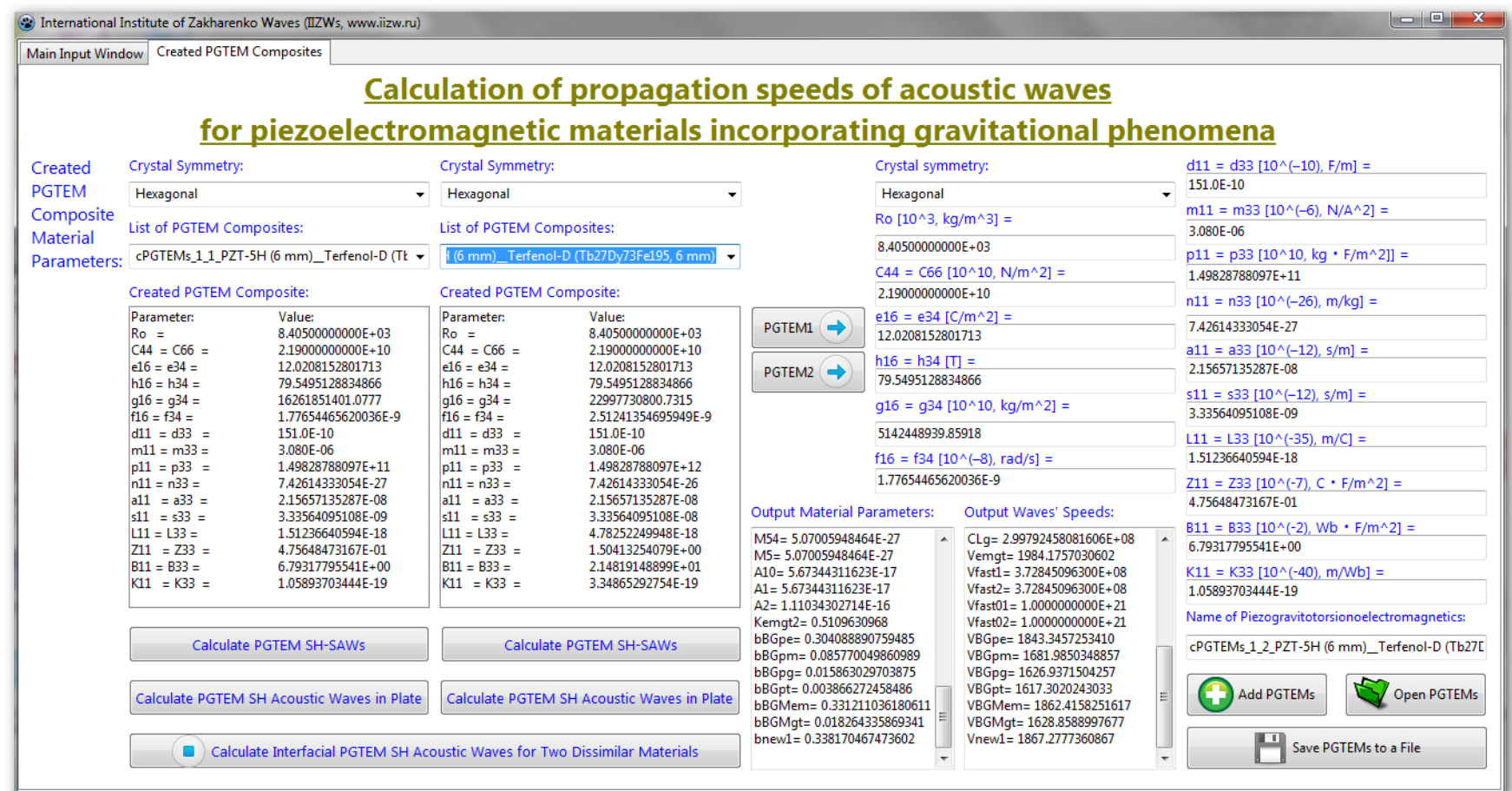

Fig. 2. The second TabSheet of the main form that contains all the input and output data of the created PGTEMs materials.

For the piezogravitotorsionoelectromagnetic (PGTEM) materials there is the second TabSheet of the main form shown in figure 2. On the left it has two dialogs for comparison of the material parameters of any pair of the PGTEM materials. Also, the left and right PGTEM materials can be moved to the right dialog for corrections of the values of the PGTEM material by pushing the buttons called "PGTEM1" and "PGTEM2" with arrows, respectively. The calculated material and wave parameters are shown in two output columns situated between two groups of buttons.
The group of three buttons on the bottom-right allows the user to add, load, and save any desired PGTEM material with the material parameters located in the fields above these buttons up to the crystal symmetry. So, the user can save the PGTEM parameters to a text file, open a text file containing the material parameters created by the user, and add the loaded (created, corrected) PGTEM parameters to the common list of the PGTEMs. The output form with figures and calculated data shown in figure 3 is created as soon as the user pushes on one of the left-group buttons. 


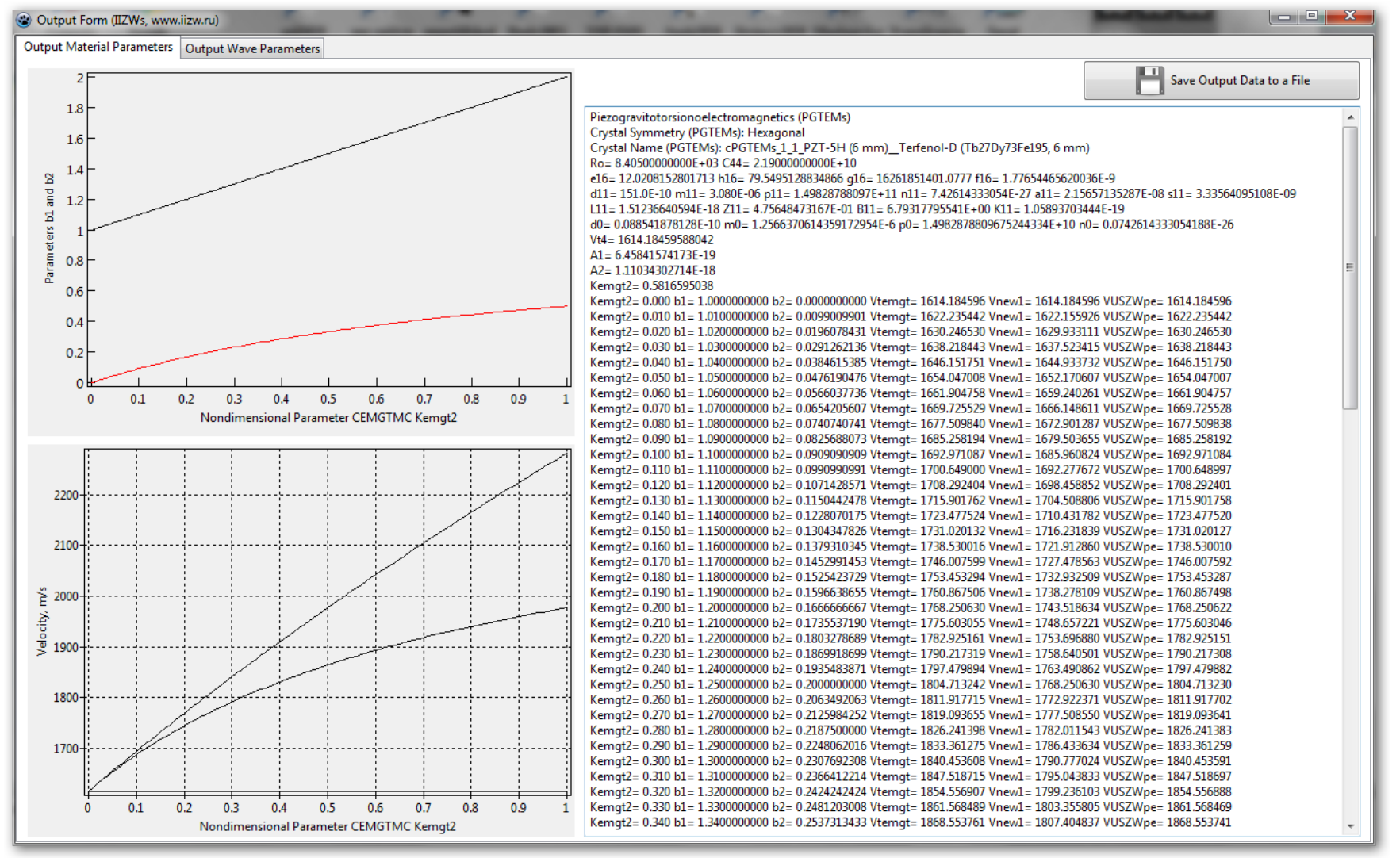

Fig. 3. The output form with figures and input and output data that is created when the user push the buton called "Calculate PGTEM SH-SAWs" located on the second TabSheet of the main form.

In the appeared output form shown in figure 3 there are two figures and several columns of output data. All these data can be saved to a file by pushing the single button on the topright. The output form has two TabSheets with output data for the PGTEM materials. The second TabSheet is not shown here due to the page limitation for the conference proceedings. The same output form, for instance, for a piezoelectrics is appeared when the user pushes the button entitled "Calculate" situated on the other form that is called when the user pushes the longest button on the bottom of the main form shown in figure 1. For piezoelectrics, however, the output form contains only the single TabSheet with the corresponding data shown in figure 3. This is due to the fact that piezoelectrics possess a small number of the input and output data in comparison with the piezoelectromagnetics and the PGTEM materials.

Pushing the button entitled "Create PEMs, PGs, PTs, PGTs" (figure 1) causes the creation of one piezoelectromagnetics, four piezogravitics, four piezotorsionics, sixteen piezogravitotorsionics, and sixteen PGTEM composite materials. They are immediately available in the corresponding listings for calculation for their properties. This procedure if the composite materials' creation is useful because the difference in the values of some material parameters can reach forty orders in this case. However, the software was successfully tested on Windows 7 and 10 platforms in various countries. The software has demonstrated its stability and correctness.

The button entitled "Show References" (figure 1) is for loading of one extra form that contains the references for the piezoelectrics, piezomagnetics, piezoelectromagnetics, etc. This form also contains the other information and a short instruction for the user. For instance, for the piezoelectrics and piezomagnetics present in the IIZWs software there are the following default crystals: piezoelectrics PZT-7A (hexagonal symmetry class $6 \mathrm{~mm})$ [10,11], PZT-5H (6 mm) [12], CdSe (6 $m m)$ [13], $\mathrm{ZnO}(6 \mathrm{~mm})$ [14], $\mathrm{Bi}_{12} \mathrm{SiO}_{20}$ (cubic symmetry class 23) [15], $\mathrm{Bi}_{12} \mathrm{GeO}_{20}$ (23) [16], $\mathrm{Bi}_{12} \mathrm{TiO}_{20}$ (23) [17], $\mathrm{Tl}_{3} \mathrm{TaSe}_{4}$ and $\mathrm{Tl}_{3} \mathrm{VS}_{4}$ (cubic Chalcogenides, 43m) [18], $\beta$ - ZnS (43m) [19], etc.; piezomagnetics $\mathrm{CoFe}_{2} \mathrm{O}_{4}(6 \mathrm{~mm})[20,21,22,23]$, Terfenol-D ( $\left.\mathrm{Tb}_{27} \mathrm{Dy}_{73} \mathrm{Fe}_{195}, 6 \mathrm{~mm}\right)$ [24], $\mathrm{CoFe}_{2} \mathrm{O}_{4}(\mathrm{~m} 3 \mathrm{~m})$ [25, 26], Alfenol ( $\left.\mathrm{Fe}_{90} \mathrm{Al}_{10}, \mathrm{~m} 3 \mathrm{~m}\right)$ [27], Galfenol $\left(\mathrm{Fe}_{81} \mathrm{Ga}_{19}, \mathrm{~m} 3 \mathrm{~m}\right)$ [28], Metglas 2605 (FeBSiC, m3m) [29], YIG $\left(\mathrm{Y}_{3} \mathrm{Fe}_{5} \mathrm{O}_{12}\right.$, $\mathrm{m} 3 \mathrm{~m})$ [30], $\mathrm{NiFe}_{2} \mathrm{O}_{4}$ and $\mathrm{Ni}(\mathrm{m} 3 \mathrm{~m})[31,32]$, etc.

It is natural that the created software can be further developed in the future concerning some improvements in the design and creation of extra output data for PGTEM materials. The theoretical developments in this research arena will also result in extra calculations of the material and wave parameters that can be added in the software.

\section{REFERENCES}

[1] A. A. Zakharenko, "On piezogravitocogravitoelectromagnetic shearhorizontal acoustic waves," Can. J. Pure Appl. Sci., vol. 10, pp. 40114028, October 2016. DOI: https://doi.org/10.5281/zenodo.1301184.

[2] A. A. Zakharenko, "The problem of finding of eigenvectors for 4P-SHSAW propagation in $6 \mathrm{~mm}$ media," Can. J. Pure Appl. Sci., vol. 11, pp. 4103-4119, February 2017. DOI: https://doi.org/10.5281/zenodo.1301202.

[3] A. A. Zakharenko, "Four independent eigenvectors and their properties for the problem of four-potential acoustic SH-wave propagation incorporating gravitational phenomena," Algebras, Groups, and Geometries, vol. 35, pp. 169-204, 2018. DOI: http://doi.org/10.5281/zenodo.3600504. 
[4] A. A. Zakharenko, "On new interfacial four-potential acoustic SHwave in dissimilar media pertaining to transversely isotropic class 6 mm," Can. J. Pure Appl. Sci., vol. 11, pp. 4321-4328, October 2017. DOI: https://doi.org/10.5281/zenodo.1301215.

[5] A. A. Zakharenko, "On existence of new dispersive four-potential SHwaves in $6 \mathrm{~mm}$ plates for new communication era based on gravitational phenomena," Can. J. Pure Appl. Sci., vol. 12, pp. 45854591, October 2018. DOI: http://doi.org/10.5281/zenodo.1471100.

[6] A. A. Zakharenko, "On separation of exchange terms for four-potential acoustic SH-wave case with dependence on gravitational parameters," Hadronic J., vol. 41, pp. 349-370, 2018. DOI: http://doi.org/10.5281/zenodo.2842082.

[7] A. A. Zakharenko, "On evaluations of fast speeds of propagation of gravitational phenomena: A review," Can. J. Pure Appl. Sci., vol. 14, pp. 4947-4963, February $2020 . \quad$ DOI: http://doi.org/10.5281/zenodo.3688779.

[8] A. A. Zakharenko, "On necessity of development of instant interplanetary telecommunication based on some gravitational phenomena for remote medical diagnostics and treatment," Can. J. Pure Appl. Sci., vol. 12, pp. 4481-4487, June 2018. DOI: https://doi.org/10.5281/zenodo.1301289.

[9] B. M. Yavorsky, A. A. Detlaf and A. K. Lebedev, The Physics Reference Book for Engineers and Students of the Higher Education, 8th edi., Moscow: ONICS Publishers, 2006, pp. 1054.

[10] J. S. Yang, "Bleustein-Gulyaev waves in piezoelectromagnetic materials," Int. J. Appl. Electromagnetics Mechanics, vol. 12, pp. 235 240, March 2000.

[11] H. Jaffe and D. A. Berlincourt, "Piezoelectric transducer materials," Proceedings of the IEEE, vol. 53, pp. 1372-1386, October 1965.

[12] Y. E. Pak, "Linear electro-elastic fracture mechanics of piezoelectric materials," Int. J. Fracture, vol. 54, pp. 79-100, January 1992.

[13] J. N. Sharma, M. Pal, D. Chand, "Propagation characteristics of Rayleigh waves in transversely isotropic piezothermoelastic materials," J. Sound Vibration, vol. 284, pp. 227-248, 2005. DOI: https://doi.org/10.1016/j.jsv.2004.06.036.

[14] J. Su, Z. B. Kuang, H. Liu, "Love wave in $\mathrm{ZnO} / \mathrm{SiO}_{2} / \mathrm{Si}$ structure with initial stresses," J. Sound Vibration, vol. 286, pp. 981- 999, 2005.

[15] V. P. Kamenov, Y. Hu, E. Shamonina, K. H. Ringhofer, V. Y. Gayvoronsky, "Two-wave mixing in (111)-cut $\mathrm{Bi}_{12} \mathrm{SiO}_{20}$ and $\mathrm{Bi}_{12} \mathrm{TiO}_{20}$ crystals: characterization and comparison with the general orientation," Phys, Rev. E, vol. 62, pp. 2863-2870, 2000. DOI: https://doi.org/10.1103/PhysRevE.62.2863.

[16] A. A. Zakharenko, Seven New SH-SAWs in Cubic Piezoelectromagnetics, Riga, Latvia: LAP LAMBERT Academic Publishing $\mathrm{GmbH} \&$ Co. KG, 2011, pp. 172. DOI: https://doi.org/10.13140/2.1.2428.0001.

[17] A. A. Zakharenko, "New solutions of shear waves in piezoelectric cubic crystals," J. Zhejiang University SCIENCE A, vol. 8, pp. 669674, 2007. DOI: https://doi.org/10.1631/jzus.2007.A0669.
[18] J. Henaff, M. Feldmann, M. A. Kirov, "Piezoelectric crystals for surface acoustic waves (Quartz, $\mathrm{LiNbO}_{3}, \mathrm{LiTaO}_{3}, \mathrm{Tl}_{3} \mathrm{VS}_{4}, \mathrm{Tl}_{3} \mathrm{TaSe}_{4}$, $\left.\mathrm{AlPO}_{4}, \mathrm{GaAs}\right)$," Ferroelectrics, vol. 42, pp. 161-185, 1982.

[19] A. A. Zakharenko, Dispersive SAWs in Layered Systems Consisting of Cubic Piezoelectrics, Riga, Latvia: LAP LAMBERT Academic Publishing GmbH \& Co. KG, 2010, pp. 72. DOI: https://doi.org/10.13140/2.1.2952.2881.

[20] Y. Y. Zhou, C. F. Lü, W. Q. Chen, "Bulk wave propagation in layered piezomagnetic/piezoelectric plates with initial stresses or interface imperfections," Composite Structures, vol. 94, pp. 2736-2745, 2012. DOI: http://dx.doi.org/10.1016/j.compstruct.2012.04.006.

[21] A. R. Annigeri, N. Ganesan, S. Swarnamani, "Free vibrations of simply supported layered and multiphase magneto-electro-elastic cylindrical shells," Smart Materials Structures, vol. 15 pp. 459-467, 2006. DOI: https://doi.org/10.1088/0964-1726/15/2/027.

[22] J. Aboudi, "Micromechanical analysis of fully coupled electromagneto-thermo-elastic multiphase composites," Smart Materials Structures, vol. 10, pp. 867-877, 2001.

[23] T. J.-Ch. Liu and Ch.-H. Chue, "On the singularities in a bimaterial magneto-electro-elastic composite wedge under antiplane deformation," Composite Structures, vol. 72, pp. 254-265, 2006. DOI: https://doi.org/10.1016/i.compstruct.2004.11.009.

[24] A. E. Giannakopoulos and A. Z. Parmaklis, "The contact problem of a circular rigid punch on piezomagnetic materials," Int. J. Solids Structures, vol. 44, pp. 4593-4612, 2007. DOI: http://dx.doi.org/10.1016/j.ijsolstr.2006.11.040.

[25] S. Srinivas, J. Y. Li, Y. C. Zhou, A. K. Soh, "The effective magnetoelectroelastic moduli of matrix-based multiferroic composites,” J. Appl. Phys., vol. 99, 043905, 2006, pp. 7.

[26] F. Ramirez, P. R. Heyliger, E. Pan, "Free vibration response of twodimensional magneto-electro-elastic laminated plates," J. Sound Vibration, vol. 292, pp. 626-644, 2006.

[27] M. Avellaneda and G. Harshe, "Magnetoelectric effect in piezoelectric/magnetostrictive multilayer (2-2) composites," J. Intelligent Material Systems Structures, vol. 5, pp. 501-513, 1994.

[28] A. A. Zakharenko, "First evidence of surface SH-wave propagation in cubic piezomagnetics," J. Electromagnetic Analysis Applications, vol. 2, pp. 287-296, 2010. DOI: https://doi.org/10.4236/jemaa.2010.25037.

[29] J. Zhai, Sh. Dong, Z. Xing, J. Li, D. Viehland, "Giant magnetoelectric effect in Metglas/polyvinylidene fluoride laminates," Appl. Phys. Lett., vol. 89, 083507 (2006).

[30] M. Fiebig, "Revival of the magnetoelectric effect," J. Phys. D: Appl. Phys., vol. 38, pp. R123-R152, 2005.

[31] A. A. Zakharenko, "A study of SH-SAW propagation in cubic piezomagnetics for utilization in smart materials," Waves Random Complex Media, vol. 22, pp. 488-504, 2012. DOI: https://doi.org/10.1080/17455030.2012.727042.

[32] Z. Kaczkowski, "Effect of a magnetic field on the piezomagnetic parameters of some magnetostrictive materials," Ultrasonics, vol. 8, pp. 239-242, 1970. 